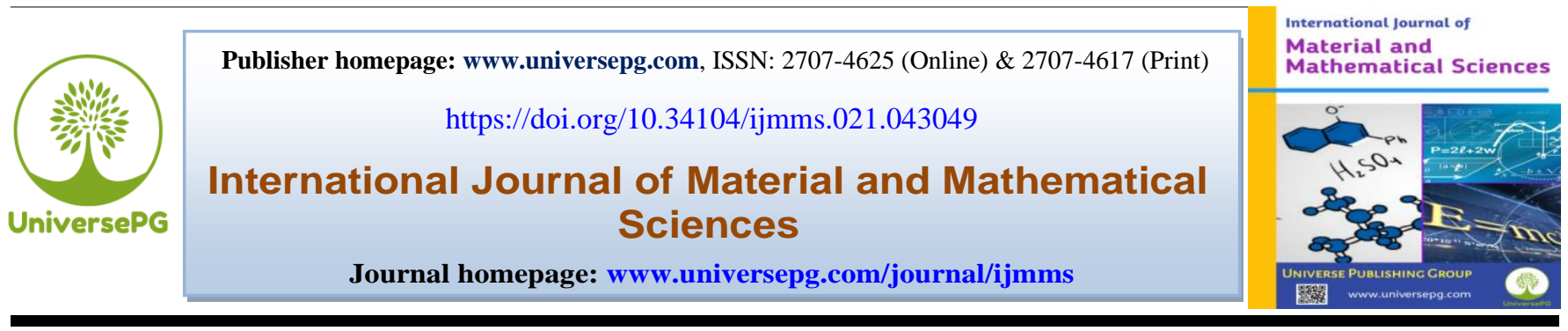

\title{
Effect of Shift Parameters in Rational Krylov Subspace Method for Solving Riccati Equations Arise from Power System Models
}

\author{
Mahtab Uddin ${ }^{1 \& 3 *}$, M. Monir Uddin ${ }^{2}$, and Md. Abdul Hakim Khan ${ }^{3}$ \\ ${ }^{1}$ Institute of Natural Sciences, United International University, Dhaka-1212, Bangladesh; ${ }^{2}$ Department of Mathematics and \\ Physics, North South University, Dhaka-1229, Bangladesh; and ${ }^{3}$ Department of Mathematics, Bangladesh University of \\ Engineering and Technology, Dhaka-1000, Bangladesh. \\ *Correspondence: mahtab@ins.uiu.ac.bd (Mahtab Uddin, Assistant Professor, Institute of Natural Sciences, United \\ International University, Dhaka-1212, Bangladesh).
}

\section{ABSTRACT}

The simulation of continuous-time algebraic Riccati equations (CARE) derived from the very large power system models is a highly laborious task and most cases infeasible due to the sophisticated structural ingredients. The computation is very time costly and the rate of convergence can be severely affected in the direct solvers. To overcome those adversities, an iterative approach Rational Krylov Subspace Method (RKSM) is introduced to deal with those large-scale CAREs. The solutions of those CAREs and hence the optimal feedback matrices can be efficiently explored by the RKSM approach to stabilize the power system models of unstable category. In this approach, shift parameters play a vital role in the convergence of the computations and size of the solution spaces. The goal of the work is to investigate the effect of different types of shift parameters on the stabilization process. To attain the mentioned objective, a modified version of the iterative RKSM algorithm is proposed by employing two types of shift parameters, namely, the adaptive ADI shifts and heuristic shifts. Qualitative discussions for the outcomes for those shift parameters are narrated by tabular and figurative methods.

Keywords: Shift parameter, RKSM, Riccati equation, Feedback stabilization, and Power system model.

\section{INTRODUCTION:}

The first-order index-1 descriptor system consisting of sparse sub-matrices is formed in a system of matrix equation having input-output relations

$$
\begin{aligned}
& E_{1} \dot{x}_{1}(t)=A_{1} x_{1}+A_{2} x_{2}+B_{1} u(t), \\
& 0=A_{3} x_{1}+A_{4} x_{2}+B_{2} u(t), \\
& y(t)=C_{1} x_{1}+C_{2} x_{2}+D u(t) \\
& x\left(t_{0}\right)=x_{0}, \quad t \geq t_{0} .
\end{aligned}
$$

In the system (1), $E_{1} \in R^{n_{1} \times n_{1}}$ is the differential coefficient matrix and $A_{1} \in R^{n_{1} \times n_{1}}, A_{2} \in R^{n_{1} \times n_{2}}$, $A_{3} \in R^{n_{2} \times n_{1}}, A_{4} \in R^{n_{2} \times n_{2}}$ are the state submatrices, respectively, whereas $B_{1} \in R^{n_{1} \times p}, B_{2} \in$ $R^{n_{2} \times p}$ are the control multiplier sub-matrices, $C_{1} \in R^{m \times n_{1}}, C_{2} \in R^{m \times n_{2}}$ are the state multiplier sub-matrices, and $D \in R^{m \times p}$ is the direct gain for $n_{1}+n_{2}=n$ with $p, m \ll n$. In the power system models, the matrix $D$ is zero or absent. Also, $x_{1}(\mathrm{t}) \in R^{n_{1}}, \quad x_{2}(\mathrm{t}) \in R^{n_{2}}$ are the state vectors, $u(t) \in R^{p}$ is the control (input), and $y(t) \in R^{m}$ is the 
output. The mentioned sub-matrices $E_{1}$ and $A_{1}$ are invertible (Hossain and Uddin, 2019).

Over the years, computational techniques are modified for the feasibility of massive systems. But computational intricacy and memory extravagant simulation tolls keep the approaches impractical. Thus, large dimensional empirical systems are needed to be reduced to smaller dimensional systems. The strategy of finding a reduced-order model (ROM) of a given system is called model order reduction (MOR), currently earning vital attention to the researchers. ROM-based techniques are extensively adopted in the technological fields of the modern scientific world (Flagg et al., 2010). An adjustable ROM size and keeping the structural design invariant of the test system is the earnest sought (Rahman et al., 2020). The mechanism of the MOR techniques needs to essentially robust maintaining minimized norm of the error.

To duly synchronize the iterative steps of the manipulation, the index-1 descriptor system (1) is to be restructured by implementing the well-known Schur-complements as

$$
\begin{aligned}
& x:=x_{1}, \\
& \mathcal{E}:=E_{1}, \\
& \mathcal{A}:=A_{1}-A_{2} A_{4}^{-1} A_{3}, \\
& \mathcal{B}:=B_{1}-A_{2} A_{4}^{-1} B_{2}, \\
& \mathcal{C}:=C_{1}-C_{2} A_{4}^{-1} A_{3}, \\
& \mathcal{D}:=D-C_{2} A_{4}^{-1} B_{2} .
\end{aligned}
$$

Then, the index-1 descriptor system (1) can be converted to an equivalent generalized LTI continuous-time system as

$$
\begin{aligned}
& \mathcal{E} \dot{x}(t)=\mathcal{A} x(t)+\mathcal{B} u(t), \\
& y(t)=\mathcal{C} x(t)+\mathcal{D} u(t) .
\end{aligned}
$$

The essence of LTI continuous-time systems is undeniable in the phenomena of the real-world engineering models, such as mechatronics, aero-nautics, and system and control theory (Rahman et al., 2021). In the analysis of system stability and its appliances, the Continuous-time Algebraic Riccati Equation (CARE) is the pivot factor (Bänsch et al., 2015). The CARE explored from (3) can be written as
$\mathcal{A}^{T} X \mathcal{E}+\mathcal{E}^{T} X \mathcal{A}-\mathcal{E}^{T} X \mathcal{B} \mathcal{B}^{T} X \mathcal{E}+\mathcal{C}^{T} \mathcal{C}=0$.

A finite solution $X$ of the CARE (4) is obtainable if no eigenvalue of the corresponding Hamiltonian matrix is purely imaginary (Abou-Kandil et al., 2012). The symmetric positive-definite solution $X$ is called stabilizing for the stable closed-loop matrix $\mathcal{A}-\mathcal{B B}^{T} X \mathcal{E}$.

Riccati-based feedback stabilization is obligate for an unstable type of system (3), and the optimal feedback matrix $K^{o}=\mathcal{B}^{T} X \mathcal{E}$ is the premier ingredient in this process (Chen and Qui, 2015). To stabilize the target system optimally, the matrix $\mathcal{A}$ is to be replaced by $\mathcal{A}_{s}=\mathcal{A}-\mathcal{B} K^{o}$. Then, the stabilized system can be formed as

$\mathcal{E} \dot{x}(t)=\mathcal{A}_{s} x(t)+\mathcal{B} u(t)$,

$y(t)=\mathcal{C} x(t)+\mathcal{D} u(t)$.

Computationally proficient solvers or analytical tools for large-scale CARE arising from the index-1 descriptors systems are still not technically cheap. Some Kleinman-Newton methods exist, which are very complicated, time laborious, and predefined structures are required (Mena and Saak, 2008). Alternating direction implicit (ADI) based approaches is computationally extravagant for the prerequisite large solution space, preconditioned initial parameters, and time-costly matrix factorization (Hasan and Uddin, 2018).

The linearization ability and enforcement of initial priories boost up the convergence rate of the simulations by the projection-based approach RKSM, which is efficiently applicable in systems with perturbations (Simoncini et al., 2013). We are extending the techniques of the RKSM approach for the standard systems that is discussed in (Simoncini, 2016). The extension is including the modification of the existing work in such a way that it can apply for index-1 descriptor systems. We will investigate the effect of the shift parameters in the RKSM algorithm and compare this for the adaptive ADI shifts and heuristic shifts, respectively.

Also, to optimally stabilize the unstable index-1 descriptor system, we will apply the Riccati-based 
feedback matrix and analyze the stabilized system through eigenspace and transient behaviors.

\section{Rational Krylov subspace method for solving index-1 descriptor systems}

If the eigenvalues of the matrix pair $(\mathcal{A}, \mathcal{E})$ satisfy $\lambda_{i}+\bar{\lambda}_{j} \neq 0$; for the all $i, j=1,2, \ldots, m$ that ensures the solution $X$ of the CARE (4) exists and unique. For a given set of competent shift parameters $\mu_{i} \in C^{+} ; i=1,2, \cdots, m$, the requisite $m-$ dimensional rational Krylov subspace can be generated to pursue the orthogonal projector $V \in$ $R^{m \times m}$. The desired rational Krylov subspace can be constructed as

$\mathcal{K}_{m}=\operatorname{span}\left(\begin{array}{c}\mathcal{C}^{T},\left(\mathcal{A}^{T}-\mu_{1} \mathcal{E}^{T}\right)^{-1} \mathcal{C}^{T} \\ \ldots \ldots, \prod_{i=1}^{m}\left(\mathcal{A}^{T}-\mu_{i} \mathcal{E}^{T}\right)^{-1} \mathcal{C}^{T}\end{array}\right)$.

Again, consider the CARE (4) and apply the Galerkin condition to it. After the simplification by matrix algebra, a low-rank CARE can be achieved as

$\widehat{\mathcal{A}}^{T} \widehat{X} \widehat{\mathcal{E}}+\widehat{\mathcal{E}}^{T} \widehat{X} \widehat{\mathcal{A}}-\widehat{\mathcal{E}}^{T} \widehat{X} \widehat{\mathcal{B}} \widehat{\mathcal{B}}^{T} \widehat{X} \widehat{\mathcal{E}}+\widehat{\mathcal{C}}^{T} \widehat{\mathcal{C}}=0$,

Here, $\widehat{X}=V^{T} X V, \widehat{\mathcal{E}}=V^{T} \mathcal{E} V, \widehat{\mathcal{A}}=V^{T} \mathcal{A} V, \widehat{\mathcal{B}}=V^{T} \mathcal{B}$, and $\widehat{\mathcal{C}}=\mathcal{C} V$.

The equation (6) is a low-rank CARE and can be solved by MATLAB care command or any existing methods, such as the Schur-decomposition method.

For the quick and smooth convergence of the proposed approach, adjustable shift selection is crucial (Druskin et al., 2010). There are many schemes to find suitable shift parameters, several approaches are given in (Moret and Popolizio, 2014; Benner et al., 2010) and references therein. In the present work, we are adopting the adaptive ADI shift and the heuristic shift parameters approach for index-1 descriptor systems.

In the iterative simulation process, to generate the feasible orthogonal projector $V$ the existing set of shift parameters must be extended within the solution space. By the solution $\widehat{X}$ of the lowrank CARE (6), the solution $X$ of the CARE (4) will be approximated as $X \approx V \widehat{X} V^{T}$.

To have a stopping condition, a suitable approximation of the residual is required. The residual of $m$-th iteration can be executed in terms of the Frobenius norm $\|.\|_{F}$ as

$\|\Re\|_{F}=\left\|S J S^{T}\right\|_{F} ; \quad J=\left[\begin{array}{ccc}0 & I & 0 \\ I & 0 & I \\ 0 & I & 0\end{array}\right]$.

Here the block upper triangular matrix $S$ is found from the QR-factorization of the matrix $U$ is defined as

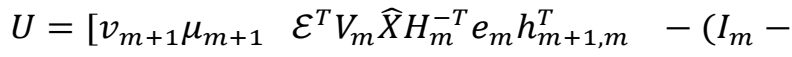
$\left.\left.V_{m} V_{m}^{T}\right) \mathcal{A}^{T} v_{m+1}\right]$.

The factor $\beta_{0}=R_{0}$ needs to be computed from the QR-factorization $\mathcal{C}^{T}=Q_{0} R_{0}$. Then, the relative residual can be estimated as

$\|\Re\|_{F}^{(\text {Relative })}=\frac{\|\Re\|_{F}}{\left\|\beta_{0}^{T} \beta_{0}\right\|_{F}}$.

The discussion about the terms and notations of the equation (8) are given in (Uddin, 2020) and the references therein.

\section{Sparsity preservation techniques}

In system (3), the matrix $\mathcal{A}$ is dense and for this reason, the rate of convergence of their structured system will be disturbed and accuracy will be affected. To avoid those inconveniences, instead of the explicit conversion of $\mathcal{A}$, a more convenient approach will be done. To find $v_{i}$, at each of the iterations, a simple linear system will be solved as

$\left(\mathcal{A}^{T}-\mu_{i} \mathcal{E}^{T}\right) v_{i}=V_{i-1}$,

$\left[\begin{array}{cc}A_{1}^{T}-\mu_{i} E_{1}^{T} & A_{3}^{T} \\ A_{2}^{T} & A_{4}^{T}\end{array}\right]\left[\begin{array}{c}v_{i} \\ \Gamma\end{array}\right]=\left[\begin{array}{c}V_{i-1} \\ 0\end{array}\right]$.

Here $\Gamma$ is the truncated term. Due to sparse system structure, the higher dimensional linear system (9) is conveniently solvable by the conventional direct solvers (Uddin, 2020). For the improvement of the consistency of the RKSM approach, explicit forms of the reduced-order matrices will not be used to construct the reduced-order system. The reduced-order matrices in sparse form can be attained in the following way

$\widehat{\mathcal{E}}:=V^{T} E_{1} V$, $\widehat{\mathcal{A}}:=V^{T} A_{1} V-\left(V^{T} A_{2}\right) A_{4}^{-1}\left(A_{3} V\right)$, 
$\widehat{\mathcal{B}}:=V^{T} B_{1}-\left(V^{T} A_{2}\right) A_{4}^{-1} B_{2}$,

$\widehat{\mathcal{C}}:=C_{1} V-C_{2} A_{4}^{-1}\left(A_{3} V\right)$.

The low-rank solution $\widehat{X}$ is symmetric and positive-definite and can be factorized as $\widehat{X}=$ $Y Y^{T}$. Consider the desired low-rank factored solution of the CARE (4) as $Z=V Y$. Thus, the solution $X$ of the (4) can be attained as $X \approx Z Z^{T}$ such that $X \approx V \widehat{X} V^{T}=(V Y)(V Y)^{T}=Z Z^{T}$.

For the future steps, the factored solution $Z=V Y$ will be stored and hence the optimal feedback matrix $K^{o}=\mathcal{B}^{T} X \mathcal{E}=\mathcal{B}^{T}\left(Z Z^{T}\right) \mathcal{E}$ can be estimated. This process is iterative and will continue until the desired convergence is achieved. The whole process is summarized in Algorithm-1.

Finally, applying $\mathcal{A}_{s}=\mathcal{A}-\mathcal{B} K^{o}$, the optimally stabilized LTI continuous-time system can be written as (5). To preserve the structure of the system, it needs to back to the original structure (1), and for this, the target system needs to be re-written as

$$
\begin{aligned}
& E_{1} \dot{x}_{1}(t)=\left(A_{1}-B_{1} K^{o}\right) x_{1}+A_{2} x_{2}+B_{1} u(t), \\
& 0=\left(A_{3}-B_{2} K^{o}\right) x_{1}+A_{4} x_{2}+B_{2} u(t), \\
& y(t)=C_{1} x_{1}+C_{2} x_{2}+D u(t) .
\end{aligned}
$$

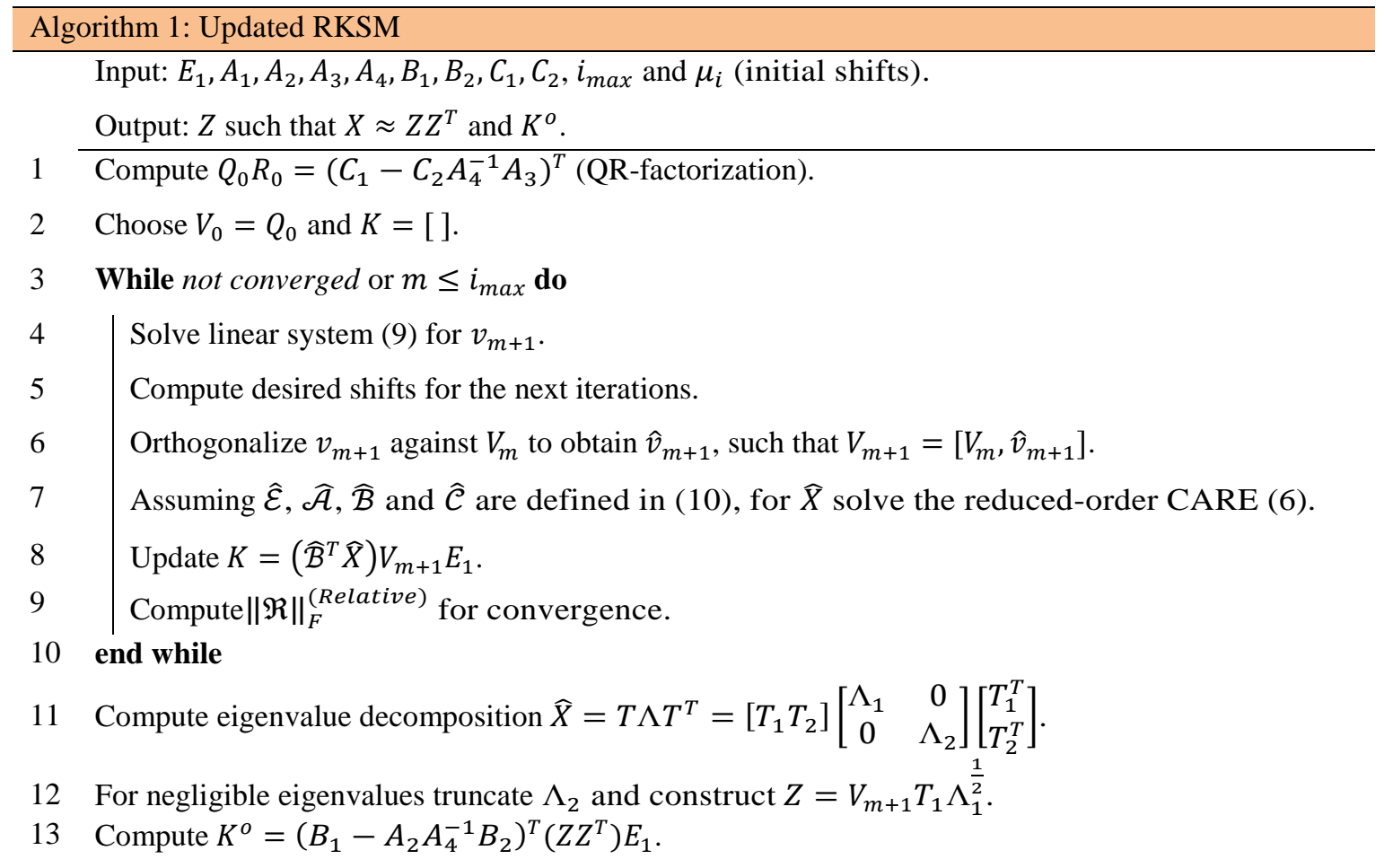

\section{Numerical results}

The eligibility and efficiency of the proposed techniques for desired shift parameters are investigated by subjecting a Brazilian Interconnected Power System (BIPS) model, namely BIPS-606 with 606 differential and 6529 algebraic variables, which is of the type unstable index-1 descriptor system (Rommes et al.,
2019; Freitas et al., 2008). The proposed techniques are applied with a tolerance level $10^{-10}$ to find low-rank approximate solutions for both adaptive ADI and heuristic shifts.

To stabilize the target model the optimal feedback matrix $K^{o}$ is used. The comparisons of several computational aspects are given in Table 1.

Table 1: Comparative analysis

\begin{tabular}{|c|c|c|c|c|c|}
\hline Shifts & Tolerance & No of iteration & Space dimension & Numerical rank & CPU time (second) \\
\cline { 1 - 4 } Adaptive ADI & \multirow{2}{*}{$10^{-10}$} & 98 & 392 & 197 & 181 \\
\cline { 1 - 4 } & & 115 & 460 & 195 & 136 \\
\hline Heuristic & & & & \\
\hline
\end{tabular}


From the above table, it is observed that by using adaptive ADI shifts a smaller solution space can be achieved, which is feasible for the memory allocation of the computational tool. In contrast, heuristic shifts are better for minimizing computational time.

The comparison of the relative residuals and the eigenvalue stabilization for the RKSM approach exerting the adaptive ADI and heuristic shifts are shown in Fig 1 and Fig 2.

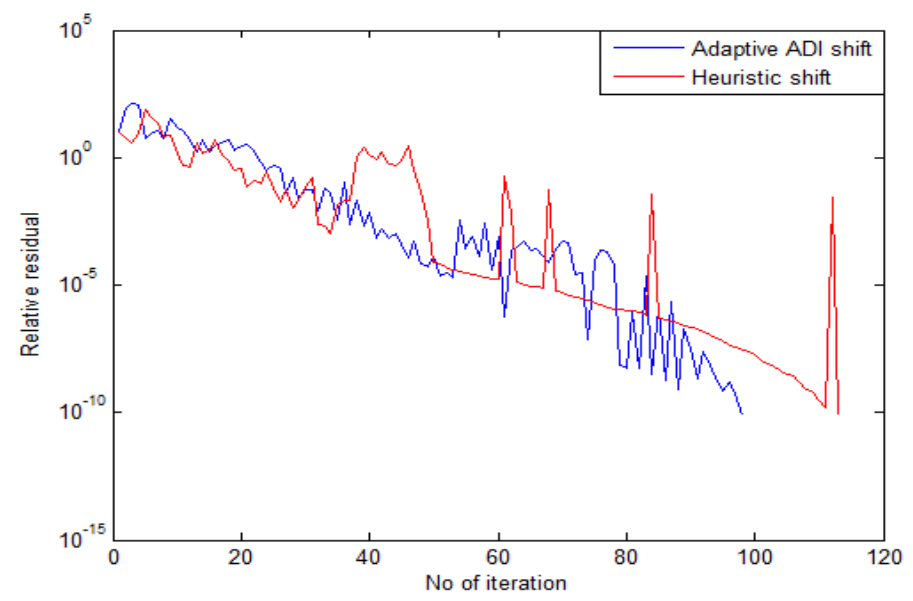

Fig 1: Comparison of the relative residuals.

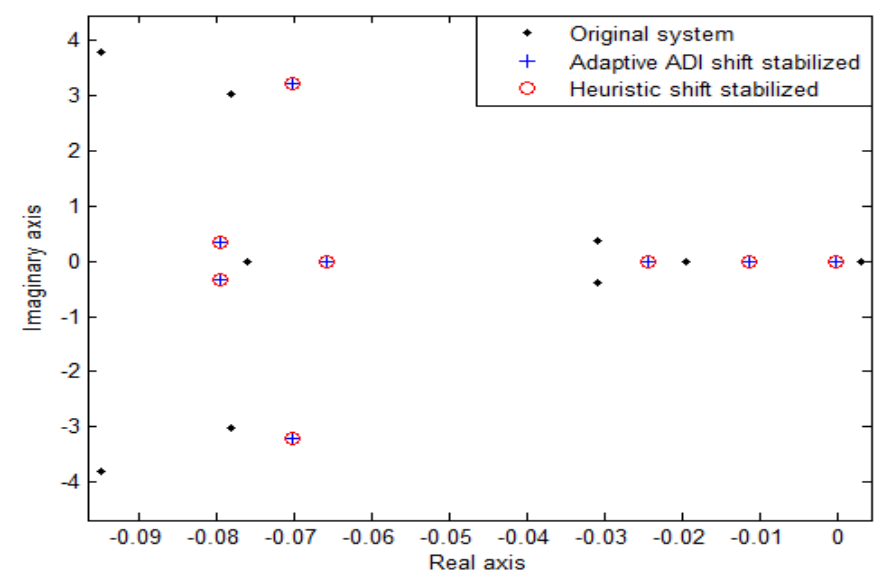

Fig 2: Comparison of the stabilization of the eigenvalues.

From the above figures, it is evident that that adaptive ADI shifts provide better relative residuals than heuristic shifts. But the stabilized eigenvalues for both of the shifts are very identical.

The sub-figures of Fig 3 depict the stabilization of the step-responses of dominant input-output relations of the target model.

UniversePG I www.universepg.com

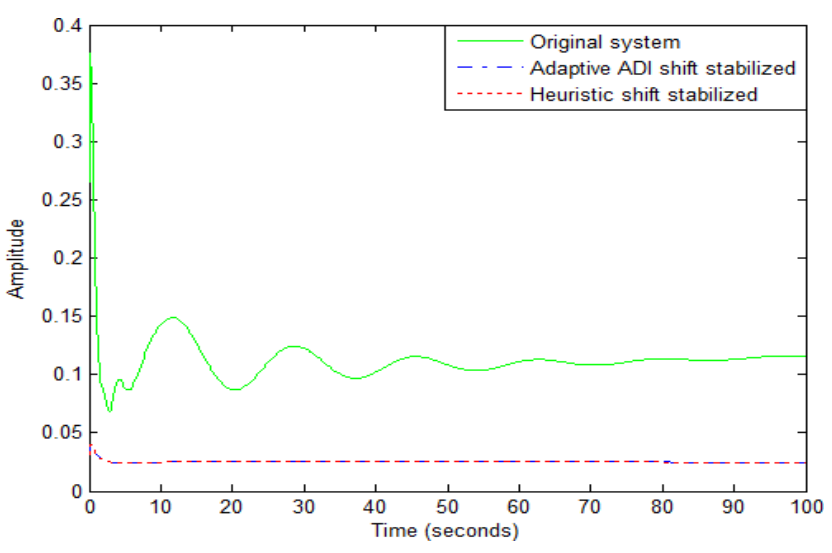

a) 1 st input/3rd output

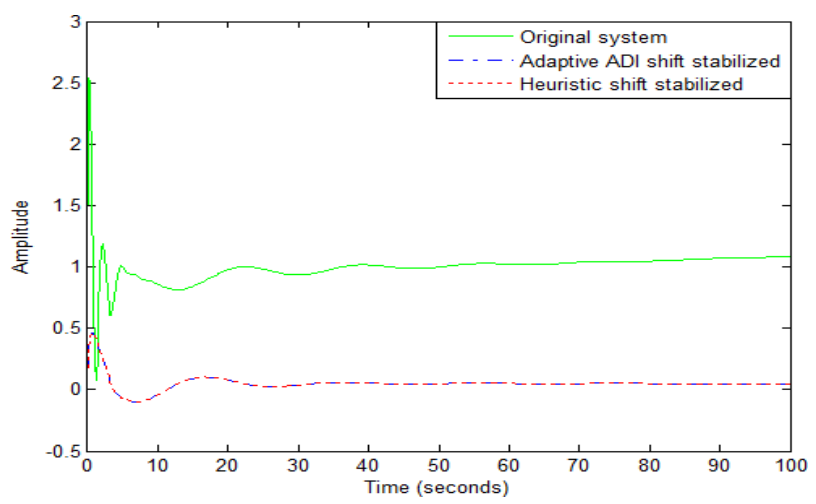

b) 2 nd input/1st output

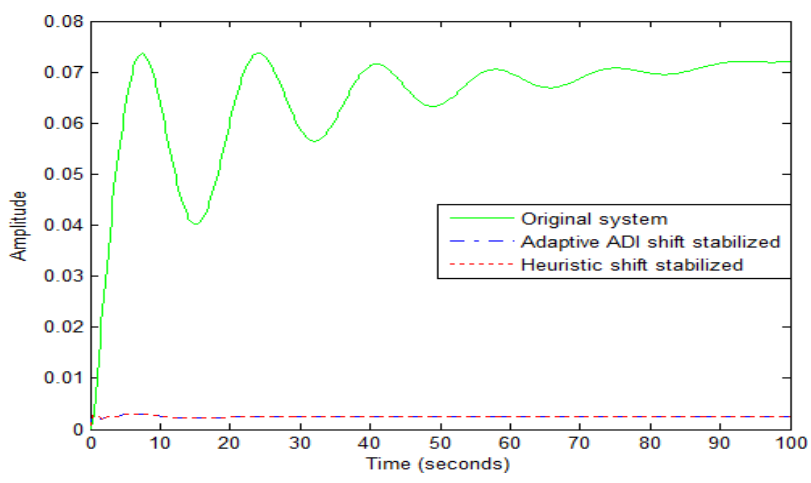

c) 3rd input/2nd output

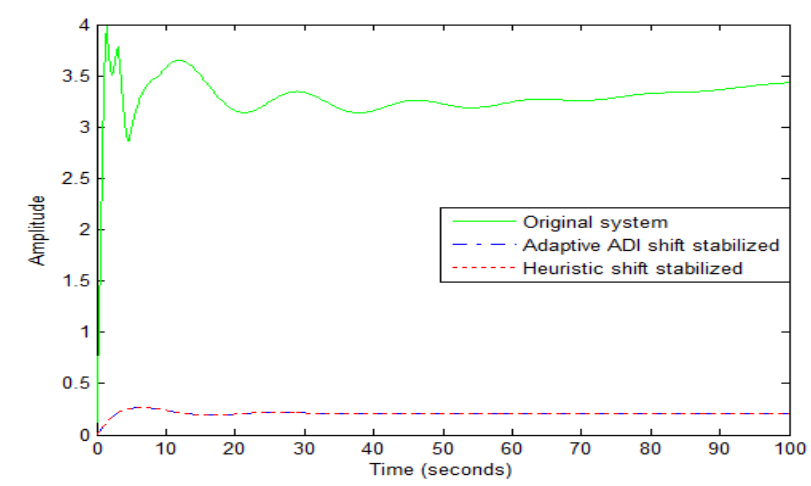

(d) 4th input/3rd output

Fig 3: Comparison of the step-responses of the ROMs. 
From the above sub-figures, it can be said that by utilizing both of the adaptive and heuristic shifts the unstable index-1 descriptor systems can be efficiently stabilized.

\section{CONCLUSION:}

In this work, the techniques to optimally stabilize the unstable index-1 descriptor system by the optimal feedback matrix attained by the solution of the Riccati equation, are introduced and embedded. To serve the purpose, the CARE corresponding to a model derived from the Brazilian Interconnected Power System has been efficiently solved by the iterative RKSM approach utilizing adaptive ADI shift and heuristic shift parameters. The robustness and time-dealing efficiency of the computation is justified by applying the techniques to the target model. From the tabular and graphical comparisons, it can be concluded that the RKSM approach utilized both the adaptive ADI and heuristic shifts, stabilized the target model with the desired efficiency. But adaptive ADI shifts are feasible for the number of iterations and space dimensions, whereas heuristic shifts are suitable for the numerical rank and computation time.

Thus, adaptive ADI shifts are applicable for the feasibility of memory allocation and heuristic shifts are comfortable for quick convergence.

\section{ACKNOWLEDGEMENT:}

We would like to make manifest our profound gratitude to Md. Motlubar Rahman, Associate Professor in Mathematics, Directorate of Secondary and Higher Education, Ministry of Education, Bangladesh for his invaluable suggestions and support in writing this article successfully.

\section{CONFLICTS OF INTEREST:}

The authors declared that there is no conflict of interest in this article.

\section{REFERENCES:}

1) Abou-Kandil H., Freiling G., Ionescu V., and Jank G. (2012). Matrix Riccati equations in control and systems theory, Birkhäuser.

2) Bänsch E., Benner P., Saak J., and Weichelt H. K. (2015). "Riccati-based boundary feedback stabilization of incompressible Navier-Stokes

UniversePG I www.universepg.com flow," SIAM Journal on Scientific Computing, 37(2), pp. A832-A858.

https://doi.org/10.1137/140980016

3) Benner P., Kürschner P., and Saak J. (2014). "Self-generating and efficient shift parameters in ADI methods for large Lyapunov and Sylvester equations," Electronic Transactions on Numerical Analysis (ETNA), 43, pp. 142https://doi.org/162. 10.17617/2.2071065

4) Chen W. and Qiu L. (2015). "Linear quadratic optimal control of continuous-time LTI systems with random input gains," IEEE Transactions on Automatic Control, 61(7), pp. 2008-2013.

5) Druskin V., Lieberman C., and Zaslavsky M. (2010). "On adaptive choice of shifts in rational Krylov subspace reduction of evolutionary problems," SIAM Journal on Scientific Computing, 32(5), pp. 2485-2496. https://doi.org/10.1137/090774082

6) Flagg G. M., Gugercin S., and Beattie C. A. (2010). "An interpolation-based approach to $H_{\text {inf }}$ model reduction of dynamical systems," in $49^{\text {th }}$ IEEE Conference on Decision and Control (CDC). IEEE, pp. 6791-6796.

7) Freitas F. D., Rommes J., and Martins N. (2008). "Gramian-based reduction method applied to large sparse power system descriptor models," IEEE Transactions on Power Systems, 23(3), pp. 1258-1270. https://doi.org/10.1109/TPWRS.2008.926693

8) Hasan S. and Uddin M. M. (2018). "Model reduction of structured dynamical systems by projecting onto the dominant eigenspace of the Gramians," Journal of Modeling and Optimization, 10(2), pp. 94-94.

https://doi.org/10.32732/jmo.2018.10.2.94

9) Hossain M. S. and Uddin M. M. (2019). "Iterative methods for solving large sparse Lyapunov equations and application to model reduction of index-1 differential-algebraic equations," Numerical Algebra, Control \& Optimization, 9(2), p. 173.

https://doi.org/10.3934/naco.2019013

10) Mena H. and Saak J. (2008). "On the parameter selection problem in the NewtonADI iteration for large-scale Riccati equa- 
tions," Electronic Transactions on Numerical Analysis, 29, pp. 136-149.

11) Moret I. and Popolizio M. (2014). "The restarted shift-and-invert Krylov method for matrix functions," Numerical Linear Algebra with Applications, 21(1), pp. 68-80.

https://onlinelibrary.wiley.com/doi/abs/10.1002/ nla. 1862

12) Rahman M. M., Uddin M. M., Andallah L. S., and Uddin M. (2020). "Interpolatory projection techniques for $\mathrm{H}_{2}$ optimal structurepreserving model-order reduction of secondorder systems," Advances in Science, Technology and Engineering Systems Journal, 5(4), pp. 715-723.

https://doi.org/10.25046/aj050485

13) Rahman M. M., Uddin M. M., Andallah L., and Uddin M. (2021). "Tangential interpolatory projections for a class of second-order in-dex-1 descriptor systems and application to Mechatronics," Production Engineering, 15(1), pp. 9-19. https://doi.org/10.1007/s11740-020-00995-4

14) Rommes J., Martins N., and Freitas F. D. (2019). "Computing rightmost eigenvalues for small-signal stability assessment of large-scale power systems," IEEE transactions on power systems, 25(2), pp. 929-938.

https://doi.org/10.1109/TPWRS.2009.2036822

15) Simoncini V., Szyld D. B., and Monsalve M. (2013). "On two numerical methods for the solution of large-scale algebraic Riccati equations," IMA J. of Numerical Analysis, 34(3), pp. 904-920.

https://doi.org/10.1093/imanum/drt015

16) Simoncini V. (2016). "Analysis of the rational Krylov subspace projection method for largescale algebraic Riccati equations," SIAM Journal on Matrix Analysis and Applications, 37(4), pp. 1655-1674. https://doi.org/10.1137/16M1059382

17) Uddin M. (2020). "Numerical study on continuous-time algebraic Riccati equations arising from large-scale sparse descriptor systems," Master's thesis, Bangladesh University of Engineering and Technology.

18) Uddin M. M. (2019). Computational Methods for Approximation of Large-scale Dynamical Systems, Chapman and Hall/CRC.

Citation: Uddin M, Uddin MM, and Khan MAH. (2021). Effect of shift parameters in rational Krylov subspace method for solving Riccati equations arise from power system models, Int. J. Mat. Math. Sci., 3(2), 43-49. https://doi.org/10.34104/ijmms.021.043049 (c) 